\title{
A Survey of Communication Technologies for the Energy Local Area Network in the Energy Internet
}

\author{
Zhongwei Sun, Weiyi Liang \\ School of Electrical \& Electronic Engineering, North China Electric Power University, Beijing, China \\ sunzwcn@aliyun.com
}

Keywords: Energy Internet, Energy Local Area Network, Smart grid, Communication Technologies. Abstract. Energy internet, the results of deep integration of the energy system and the internet, is an important infrastructure to solve high-permeability of renewable energy generation in the future grid. Energy Local Area Network (ELAN), a network consisting of an energy router and its attached multiple end-users, plays an extremely important role in the energy internet. This paper focuses on the communication technologies for the ELAN. More specifically, the representative communication technologies adopted for home area networks (HANs) and field area networks/neighborhood area networks (FANs/NANs) are discussed.

\section{Introduction}

The utility industry across the world is trying to address numerous challenges that occur in exiting electrical grid, including generation diversification, optimal deployment of expensive assets, demand response, energy conservation, and reduction of the industry's overall carbon footprint. It is evident that such critical issues cannot be addressed within the confines of the existing electricity grid.

The Smart Grid (SG) is expected to address the major shortcomings of the existing grid. In essence, the smart grid is the modernization of the exiting electric grid for the purpose of enabling bidirectional flows of information and electricity in order to achieve various goals [1]. As commonly cited in the literatures, the smart grid possess the following attributes: a) It is self-healing from power disturbance events; b) It enables active participation by consumers in demand response; c) It operates resiliently against both physical and cyber attacks; d) It provides quality power that meets 21 st century needs; e) It accommodates all generation and storage options.

While SG is focusing on the informization and intellectualization of the existing power grid, the new concept of an energy internet is proposed as an internet-style solution for energy issues by integrating information and power flows bi-directionally, which can be considered as the version 2.0 of smart grids [2].

Finding the best communication technology for the energy local area network in the energy internet is not straight forward, as the communication network for the energy local area network has to support information exchange among a large number of smart meters, intelligent electronic devices, sensors and actors without or very limited human intervention. Hence, our main focus in this paper is to investigate the communication technologies for the Energy Local Area Network (ELAN) in the energy internet.

\section{Overview of Energy Internet}

The large-scale exploitation and utilization of renewable energy will become a major global energy development trends. How to implement extensive interconnection of distributed renewable energy and how to increase energy efficiency and flexibility of the energy system are key problems. Energy internet, the results of integration of the energy system and the internet, is an important infrastructure to solve high-permeability of renewable energy generation in the future grid. The energy internet is a new focus of concern in current international academic and industrial areas, and a lot of research achievements have been made. 
Large scale integration of Distributed Renewable Energy Resources (DRERs), Distributed Energy Storage Devices (DESDs) and emerging DC loads require a revolutionary paradigm shift from the legacy grid to the Smart Grid. The Future Renewable Electric Energy Delivery and Management (FREEDM) Systems Center is a National Science Foundation (NSF) Generation-III Engineering Research Center established in 2008, with the mission to develop the fundamental and enabling technologies necessary for a new and paradigm shifting power grid infrastructure, the FREEDM System. Inspired by the development of information Internet, the FREEDM center has developed "Energy Internet" concept, which may eventually shift the power and energy industry from the currently centralized mainframes to a client-based, distributed power infrastructure, such as to accommodate the myriad of DRERs, DESDs, and emerging DC loads, further allowing the customers to exchange energy freely and reliably [3].

Pervasive information can fundamentally change how electrical power is produced, distributed and used. The crucial insight is to integrate information exchange everywhere that power is transferred. Katz et al have presented an information-centric energy infrastructure [4]. An Information-centered Energy Network is an information overlay on the energy distribution system in its various physical manifestations, e.g., machine rooms, buildings, neighborhoods, isolated generation islands and regional grids. A schematic view of such an overlay network is presented in the paper. Collections of supplies or loads or storage capabilities can be grouped into energy subnets that interact with other parts of the network through well defined interfaces, called an Intelligent Power Switch (IPS) in the figure. It serves to represent the aggregate demand and supply of its constituents as a schedule commodity that can be forecasted, resourced and adapted. It provides the communication exchange that accompanies the on-going energy transactions between supplies and loads.

The State Grid Corporation of China, one of the world's biggest utility companies, is pushing to build a global energy network. According to [5], the key to global energy interconnectivity was an ultra high voltage power grid, with a smart grid as the foundation and clean energy as the principle.

The Energy internet is emerging as a convergence of information technology and communication technology with power system engineering. It provides innovative concept and envision for enhancing the capability of electrical grid in form of internet style power generation, power distribution, energy storage, grid monitoring and communication. When the current energy infrastructure is fused in an open and peer-to-peer internet-style way, the whole energy system will enter the ideal state of balance, which contains the power output balance for energy utilities and the consumption balance for users.

\section{Basic Architecture of Energy Internet}

Energy Internet may be the single, worldwide cyber-energy network that interconnects with other cyber-energy networks through energy routers in which end-users can exchange the energy flexibly. Two critical elements in the Energy Internet are intelligent end-user and energy router [6]. Intelligent end-user is a machine that can be programmed to manipulate the generation, storage or consumption of the power energy, and to communicate with others regarding energy exchange. The intelligent end users can be divided into three major types: DRERs, DESDs, and loads. The energy router is core of the energy internet. It has power management functionalities which routes generated and/or stored energy to different location based on customers' set priorities. The total energy collected can be divided among the locations such as for local loads (self-use), battery storage and feed-in to the grid..

The concept of energy local area network is extremely important in the energy internet. The concept of Energy Local Area Network (ELAN) is a demand-based autonomous entity consisting of an appropriated mixture of different customers, charged with the necessary authority to maintain its own health by regulating or curtailing the power consumption of its members. Once all ELANs have achieved security, the whole grid, which is constructed by a number of ELANs in a hierarchical manner, achieves security as well. A network consisting of an energy router and its attached multiple end-users is referred as an ELAN, as shown in Fig. 1. Further, several ELANs can be configured as an 
Energy Wide Area Network (EWAN) for which the energy routers, can exchange energy between the attached end-users in the ELAN over the Energy Internet.

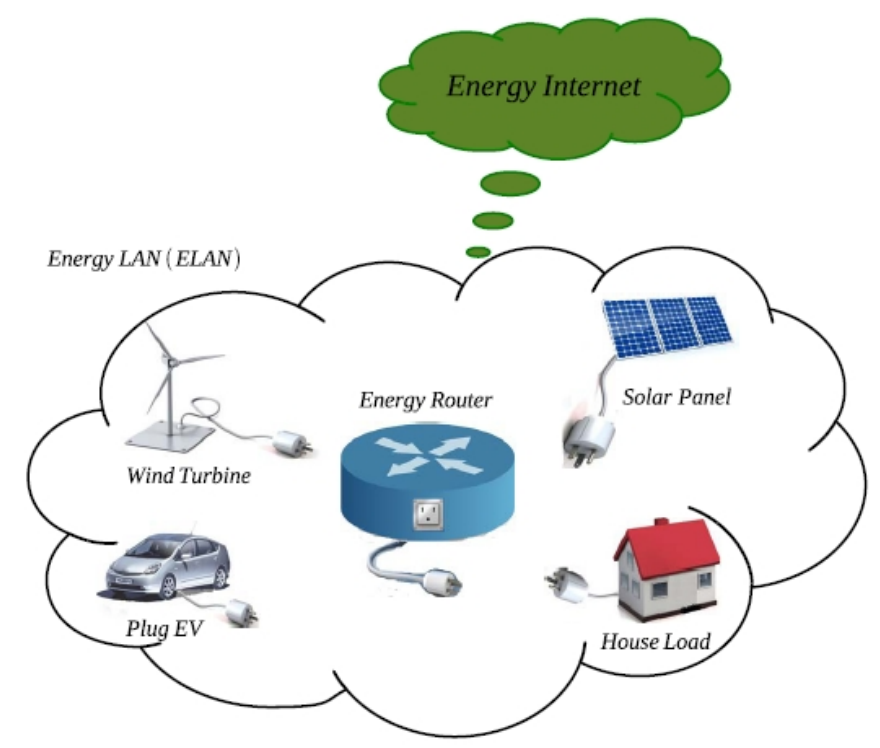

Fig. 1. Energy Internet: End Users, Energy Routers, and ELAN [6].

\section{Enabling Communication Technologies for ELAN}

The smart grid is a modern electric power grid infrastructure for improved efficiency, reliability and safety, with smooth integration of renewable and alternative energy sources, through automated control and modern communications technologies. As shown in Fig. 2, the overall architecture of the SG can be decomposed into the power system layer and communication layer. The power system layer is an integration of various electrical power generation systems, power transmission and distribution grids, substations, customers, and so on. The communication layer can be divided into three segments, i.e. Home Area Networks (HANs), Field Area Network (FANs)/Neighborhood Area Networks (NANs) and Wide Area Networks (WANs). An illustrative architecture of smart grid from generation to consumer sides is shown in Fig. 1. ELAN generally covers the area of a power distribution and customer domains. The FANs/NANs and HANs in the smart grid is considered in this paper.

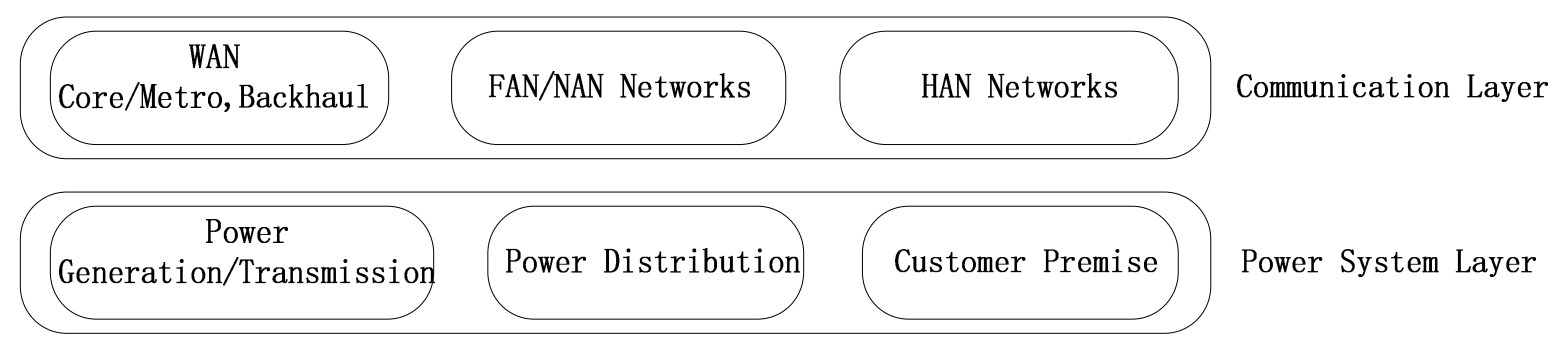

Fig. 2 The overall layered architecture of SG

HANs Communication. The Association of Home Appliance Manufacturers has conducted research on the communication technologies that best meet the requirements of home-smart appliances. This research has shown that Wireless Local Area Networks (WLANs), Wireless Sensor Networks (WSNs) such as Zigbee, and Power Line Communications (PLCs) such as HomePlug are the candidates for the HAN category. The wireless communications in HAN is preferred over wired since it allows flexible addition and removal of devices and reduces installation costs. Furthermore, the sheer volume of home automation networks with high node density may make wired approaches impractical. 
NANs/FANs Communication. Possible protocols/standards for NANs could be based on wireless or wired technologies. On the wireless side, IEEE 802.11s, RF Mesh, Worldwide Interoperability for Microwave Access (WiMAX) and cellular standards, such as 3G, 4G are some of the stronger candidates. On the wired side, ethernet, broadband over power lines and passive optical networks are possible options to use. Some electric utilities prefer fiber optic cables to have low latency and superior communication performance, others prefer WiMAX where cellular and RF mesh technologies do not have coverage over the area.

\section{Conclusion}

Energy internet is an important infrastructure to solve high-permeability of renewable energy generation in the future grid, and ELAN plays an extremely important role in the energy internet. In this paper, the communication technologies for ELAN are investigated. Since the ELAN cover the area of power distribution domain and premise domain, the representative communication technologies for the HANs and the FAN/NAN are discussed.

\section{References}

[1] X. Fang, S. Misra, G. Xue, D. Yang: Smart grid - the new and improved power grid: a survey. IEEE Communications Surveys Tutorials, Vol. 14 (2011), p.944-980.

[2] J. Cao and M. Yang: Energy Internet - Towards Smart Grid 2.0. In: Fourth International Conference on Networking and Distributed Computing, 2013.

[3] A. Q. HUANG et al: The Future Renewable Electric Energy Delivery and Management (FREEDM) System: The Energy Internet. Proc the IEEE, 2011,12(17): p133-148.

[4] R. H. Katz et al: An information-centric energy infrastructure: The Berkeley view. Sustainable Computing: Informatics and Systems. 2011,1(1): p7-22.

[5] Zhenya Liu: Global energy internet [M]. Beijing: China Electric Power Press, 2015.

[6] J. Zhang, W. Wang, and S. Bhattacharya: Architecture of Solid State Transformer-based Energy Router and Models of Energy Traffic. IEEE, 2011. 